Fludrocortisone- a treatment for tubulopathy post paediatric renal transplantation: A national paediatric nephrology unit experience

Ali SR ${ }^{1}$, Shaheen $I^{1}$, Young $D^{2}$, Ramage ${ }^{1}$, Maxwell $H^{1}$, Hughes DA $^{1}$, Athavale $D^{1}$, Shaikh $\mathrm{MG}^{3}$

1. Department of Paediatric Nephrology, Royal Hospital for Children, 1345 Govan Road, Glasgow, UK, G51 4TF.

2. Department of Mathematics and Statistics, University of Strathclyde, 16 Richmond Street, Glasgow, UK, G1 1XQ.

3. Department of Paediatric Endocrinology and Diabetes, Royal Hospital for Children, 1345 Govan Road, Glasgow, UK, G51 4TF.

\title{
Correspondence
}

Dr MG Shaikh

Department of Paediatric Endocrinology and Diabetes, Royal Hospital for Children, 1345 Govan Road, Glasgow, UK. G51 4TF

Email: guftar.shaikh@nhs.net

Tel: 01414516548 


\section{Fludrocortisone- a treatment for tubulopathy post paediatric renal transplantation: A national paediatric nephrology unit experience}

Ali SR, Shaheen I, Young D, Ramage I, Maxwell H, Hughes DA, Athavale D, Shaikh MG

Pediatr Transplantation 2017

\section{ABSTRACT}

Background Calcineurin inhibitors post renal transplantation are recognised to cause tubulopathies in the form of hyponatremia, hyperkalemia and acidosis. Sodium supplementation may be required, increasing medication burden and potentially resulting in poor compliance. Fludrocortisone has been beneficial in addressing tubulopathies in adult studies, with limited paediatric data available. Methods A retrospective review of data from an electronic renal database from December 2014 to January 2016. Results 47 post-transplant patients were reviewed with 23 (49\%) patients on sodium chloride or bicarbonate. 9 patients, aged 8.3 years (range 4.916.4) commenced fludrocortisone 22 months (range 1-80) after transplant and were followed up for 9 months (range 2-20). All patients stopped sodium bicarbonate; all had a reduction or no increase in total daily doses of sodium chloride. Potassium levels were significantly lower on fludrocortisone, 5.2 vs $4.5 \mathrm{mmol} / \mathrm{l}, \mathrm{p}=0.04$. No difference was noted in renal function (eGFR 77.8 vs $81.7 \mathrm{ml} / \mathrm{min} / 1.73 \mathrm{~m} 2, \mathrm{p}=0.45$ ) and no significant increase in systolic blood pressure (z-scores 0.99 vs $0.85, p=0.92$ ). No side effects secondary to treatment with fludrocortisone were reported. Conclusions A significant proportion of renal transplant patients were on sodium supplementation. Fludrocortisone reduced sodium supplementation without significant effects on renal function or blood pressure. Fludrocortisone appears to be safe and effective for tubulopathies in children post transplantation. 


\section{Keywords}

Bicarbonate, Chloride, Fludrocortisone, Pediatrics, Renal transplantation, Renal replacement therapy, Sodium, Tubulopathy 


\section{INTRODUCTION}

Tubulopathy, in the form of hyperkalaemia, metabolic acidosis and salt-wasting syndromes, has been recognised in the literature, post transplantation ${ }^{1,2}$. However, the incidence of this type 4 renal tubular acidosis remains unknown. Whilst tubular dysfunction can occur secondary to renal impairment and interstitial damage from acute episodes and nephrotoxic drugs $^{3}$; immunosuppressive regimens, namely calcineurin inhibitors (CNI) (Ciclosporin, Tacrolimus) have also been directly implicated ${ }^{4,5,6}$. These drugs are thought to lead to a down regulation of the mineralocorticoid receptors and a resultant pseudohypoaldosteronism picture with normal aldosterone levels. There is perhaps a suggestion that tubulopathy is more associated with tacrolimus than ciclosporin ${ }^{5}$ with transplant immunosuppressive regimens now more likely to use the former.

Fludrocortisone, a corticosteroid with high glucocorticoid and potent mineralocorticoid activity, has been shown to be beneficial when used in adults with end-stage renal disease (ESRD) who are anuric, on haemodialysis and hyperkalaemic ${ }^{7}$. In managing $\mathrm{CNI}$ associated hyperkalaemia, adult literature reports a small number of cases with a beneficial response to fludrocortisone, and emphasises the benefits of fludrocortisone as a safe and effective treatment in reversing post transplant tubulopathies with normalisation of electrolytes ${ }^{8,9,10}$.

Tubulopathy when occurring post transplantation requires the initiation of electrolyte supplementation, particularly sodium bicarbonate and sodium chloride replacement, to aid amongst other factors, growth. However, this adds to the medication burden, potentially therefore affecting compliance ${ }^{11}$.

Paediatric data remains limited with only one centre reporting the efficacy of fludrocortisone in a paediatric population for management of tubulopathy post renal transplantation $^{12}$. We report our national experience of the efficacy of fludrocortisone 
in the management of post renal transplant tubulopathy and its potential benefits in paediatric renal transplantation.

\section{MATERIALS AND METHODS}

A retrospective case review of data collected from a multi-centre, electronic renal patient database (Strathclyde Electronic Renal Patient Record, SERPR) was undertaken over a 14 month time period from December 2014 to January 2016. Data was collected for all paediatric renal transplant recipients reviewed in the tertiary paediatric renal service during the study period.

Patient demographics, medication, dietary regimens and renal function data was collected for all patients who were commenced on sodium supplements and subsequently fludrocortisone post renal transplantation. Patients were commenced on an initial total daily dose of between $25-50$ micrograms of fludrocortisone with two patients commenced on a starting dose of 100 micrograms (range 0.8-4.0 $\mu \mathrm{g} / \mathrm{kg} / \mathrm{day})$. Dose increment adjustments of 25-50 micrograms were made depending on tolerance, biochemistry results, concurrent electrolyte supplementation and blood pressure measurements. Maximum dose ranges from 75 to 150 micrograms, were used for all patients other than one patient who had a maximum dose of 200 micrograms at follow-up (range 2.9-8.1 $\mu \mathrm{g} / \mathrm{kg} /$ day). All patients were given a once daily oral preparation of fludrocortisone. Blood pressure measurements were referenced as per standard accepted $4^{\text {th }}$ Task Force Report data ${ }^{13}$.

Non-parametric data was analysed using paired t-tests to compare pretreatment and treatment values (on fludrocortisone). The results are presented as median and range intervals.

Ethics approval was not required as this was a retrospective case-series corresponding to service audit. 


\section{RESULTS}

\section{Clinical and demographic data}

Forty-seven (35 males and 12 females) paediatric renal transplant recipients were identified from the SERPR database and reviewed in the tertiary paediatric renal service during the study period. 23 (17 males) patients (48.9\%) were commenced on sodium supplementation (sodium chloride, sodium bicarbonate). Of the cohort of patients commenced on sodium supplementation, 6 were also commenced on fludrocortisone. A further 3 patients were commenced directly onto fludrocortisone to directly manage tubular dysfunction post transplantation. A total of 9 patients received fludrocortisone (Table 1). Two of the 3 patients commenced directly onto fludrocortisone had previously been on sodium supplementation but not during the study period.

The median age of patients commenced on fludrocortisone was 8.3 years (range 4.9-16.4). Fludrocortisone was given at a median of 22 months (range 1-80) after renal transplantation. Patients were followed up for a median of 9 months (range 2-20) after commencing fludrocortisone, this was equal to the time from commencing fludrocortisone to attaining the maximum dose of fludrocortisone.

All 9 patients were taking tacrolimus post transplantation, with a median tacrolimus trough level of $6.7 \mathrm{ng} / \mathrm{ml}$ (range 2.7-10.4). Five patients (Table 1, Patients 3-6, 7) were additionally taking mycophenolate mofetil (MMF) post transplant. No patients were on a maintenance steroid medication regimen.

\section{Effect on sodium supplementation}

At follow-up, all patients commenced on fludrocortisone therapy were able to discontinue sodium bicarbonate supplementation and either had a reduction or no 
increase in the total daily dose of sodium chloride (Table 1). In the 3 patients who commenced fludrocortisone prior to electrolyte supplementation, sodium and bicarbonate values stabilised. Pre-treatment sodium levels (mmol/l) were 132, 134 and 132 and on the maximum dose of fludrocortisone, post-treatment were 138, 141 and 137. Pre-treatment bicarbonate levels $(\mathrm{mmol} / \mathrm{l})$ were $21,18,16$ and posttreatment levels were 23, 22 and 21.

\section{Effect on renal function, serum potassium and blood pressure}

There was no significant change in renal function. The median eGFR (calculated using the Schwartz formula ${ }^{14}$ ) pre-treatment was 77.8 compared to 81.7 $\mathrm{ml} / \mathrm{min} / 1.73 \mathrm{~m}^{2}$ on the maximum dose of fludrocortisone, $\mathrm{p}=0.45$ (Fig. 1a).

Serum potassium levels (Fig. 1b) were significantly lower following treatment with fludrocortisone, 5.2 compared to $4.5 \mathrm{mmol} / \mathrm{l}, \mathrm{p}=0.04$. Two patients had received specific dietetic advice on maintaining a low potassium diet prior to commencing fludrocortisone. One patient in this cohort had a strict potassium restricted diet; this patient was not on electrolyte supplementation prior to treatment with fludrocortisone.

There was no significant effect on blood pressure following treatment with fludrocortisone, systolic BP 107 compared to $106 \mathrm{mmHg}, \mathrm{p}=0.81$; diastolic BP 68 compared to $66 \mathrm{mmHg}, \mathrm{p}=0.37$. In addition, there was no significant difference in median systolic blood pressure z-scores (based on age, height and gender) before and whilst on fludrocortisone, 0.99 vs $0.85, p=0.92$ (Fig. 1c). Three patients were taking amlodipine as an antihypertensive agent prior to commencing fludrocortisone. Of these, one patient had commenced amlodipine one month prior to fludrocortisone and two patients had started amlodipine one year prior to treatment with fludrocortisone. All patients' antihypertensive doses remained unchanged at followup. 
There were no side effects of treatment with fludrocortisone reported in this cohort, requiring cessation of therapy.

\section{DISCUSSION}

There remains an ongoing debate regarding the development of tubulopathies post renal transplantation with perhaps under recognition in clinical practice. Acute nephrotoxic injuries certainly contribute in many cases, however, $\mathrm{CNI}$ based immunosuppressive regimens are certainly recognised to cause tubular dysfunction ${ }^{2,4,5,6}$. Renal transplant patients treated with CNIs such as ciclosporin A and tacrolimus (FK 506) may develop signs of partial resistance to aldosterone action with subsequent hyperkalaemia and metabolic acidosis ${ }^{2}$, thus, partially explaining the mechanism of action of fludrocortisone in this setting. These electrolyte imbalances are believed to be the result of direct inhibitory effects on the ion-exchange systems of the distal tubule of the nephron such as the Na-K-ATPase and $\mathrm{Na}-\mathrm{K}-2 \mathrm{Cl}$ co-transporters. Despite normal aldosterone levels in these patients, increased inactive renin levels in transplant recipients suggest a disturbance with the Renin-Angiotensin-Aldosterone System. Heering et $\mathrm{al}^{4}$ described aldosterone resistance presenting as hyperkalaemia and metabolic acidosis post transplantation with ciclosporin and a potential association with downregulation of mineralocorticoid receptor expression on peripheral leukocytes.

Fludrocortisone, with its dual glucocorticoid and mineralocorticoid role, acts via the Na-K pump in the renal tubule. The intracellular shift of potassium may play a role in the potassium lowering effect of fludrocortisone. The use of fludrocortisone as a treatment for tubulopathies post paediatric renal transplantation remains a novel approach. The retrospective case series of 15 paediatric patients by Bacchetta et $\mathrm{al}^{12}$ reported a significant reduction in sodium supplementation following treatment with fludrocortisone. However, a significant increase in blood pressure was also noted in 
this cohort and almost half (47\%) were receiving anti-hypertensives prior to commencing fludrocortisone. Thus, it may be speculated that their cohort was at 'high risk' of hypertension prior to treatment with fludrocortisone. In addition, the prevailing immunosuppressive regimen was not clear in their cohort, including the presence of a steroid based regimen, which may have contributed to the increase in blood pressure observed. The primary renal diagnoses in their cohort were not dissimilar from that in our study. Furthermore, Bacchetta et al reported that 6 patients (40\%) required cessation of treatment due to side effects of fludrocortisone (including hypertension, gastric pain or hypokalaemia), parental decision or non-compliance. The initial fludrocortisone dosing regimens in this study and that by Bacchetta et al are similar. However, Bacchetta et al report using a higher dosing increment of up to $11.7 \mu \mathrm{g} / \mathrm{kg} / \mathrm{day}$ in their cohort, which may account for the increased side effect profile in their study.

Due to the large range of tacrolimus levels and small numbers in our cohort, we were unable to determine whether tubular $\mathrm{CNI}$ related adverse effects were dose related. However, our study noted a reduction in electrolyte supplementation, a reduction in serum potassium levels with no change in renal function and no change in blood pressure at follow-up. The hypokalaemic properties of fludrocortisone are an added benefit as patients on a potassium restricted diet were able to liberalise their dietary potassium intake.

Maintenance steroids are variably used in post renal transplant immunosuppressive regimens. This regimen even with the inclusion of tacrolimus may partially mask tubular dysfunction in post renal transplant populations.

Our study has demonstrated that fludrocortisone is well tolerated, with no side effects occurring secondary to treatment in our cohort. The insignificant differences in eGFR and blood pressure are unlikely to be due to the short interval as some patients were followed up for up to 20 months. However, a longer period of follow-up would be ideal and on going follow-up is still required to monitor for long-term side 
effects. It is important to be mindful of the side effect profile of fludrocortisone which may include hypertension, oedema secondary to salt and water retention, hypokalaemia and gastric upset and patients should be counseled appropriately prior to commencing treatment.

Limitations of our study include a small patient cohort. Furthermore, due to limited experience in the use of fludrocortisone in the management of tubulopathy post paediatric renal transplantation, there is currently no guidance with regards to an optimum dosing regimen in this setting. We used a starting dose of 25 to 50 micrograms of fludrocortisone, a dose similar to that used in children with salt wasting endocrinopathies ${ }^{15}$. This study needs to be replicated in larger cohorts with varying dosing regimens in order to minimise the potential side effect profile whilst at the same time maximising the efficacy of treatment with fludrocortisone. The analysis of additional data parameters including height and weight which are routinely collected in the clinical setting may also be useful in future studies to determine the potential effects of fludrocortisone on growth and development in children. Measurements of plasma renin and aldosterone may also be useful in future studies. In summary, tubulopathy in children post renal transplantation can be managed by fludrocortisone, which has been shown to reduce the requirement for electrolyte supplementation. Additionally, in our study, as fludrocortisone did not show a significant effect on renal function or blood pressure, it suggests it is a safe and effective treatment option for this indication. This study adds to the limited evidence in the literature regarding the benefits of fludrocortisone in the management of tubulopathy post renal transplantation. 
Table 1. Patients commenced on fludrocortisone

\begin{tabular}{|c|l|c|c|c|c|c|c|}
\hline \multirow{2}{*}{ Patient } & \multicolumn{1}{|c|}{ Renal diagnosis } & \multicolumn{2}{|c|}{$\begin{array}{c}\text { Fludrocortisone } \\
\text { (mcg) }\end{array}$} & \multicolumn{2}{|c|}{ NaHCO } & \multicolumn{2}{c|}{ NaCl } \\
\cline { 2 - 8 } & & Initial & Max & Pre- & With & Pre- & With \\
& & dose & dose & Fludro & Fludro & Fludro & Fludro \\
\hline 1 & Prune belly syndrome & 25 & 100 & 1.9 & 0 & 2.7 & 1.8 \\
2 & Posterior urethral valves & 25 & 150 & 2.0 & 0 & 1.8 & 0.9 \\
3 & Prune belly syndrome & 25 & 150 & 6.7 & 0 & 4.0 & 0 \\
4 & Congenital nephrotic syndrome & 50 & 75 & 3.0 & 0 & 1.8 & 1.8 \\
5 & Congenital renal hypoplasia & 50 & 150 & 5.0 & 0 & 4.5 & 4.0 \\
6 & Congenital nephrotic syndrome & 50 & 100 & 1.5 & 0 & 1.2 & 0.6 \\
\hline 7 & Congenital renal hypoplasia & 50 & 100 & - & - & - & - \\
8 & Congenital renal hypoplasia & 100 & 200 & - & - & - & - \\
9 & Chronic kidney disease stage 4 & 100 & 100 & - & - & - & - \\
\hline
\end{tabular}

$\mathrm{NaHCO}_{3}$, sodium bicarbonate; $\mathrm{NaCl}$, sodium chloride; mcg, micrograms; g, grams.

Patients 1-6 were taking sodium supplements prior to commencing fludrocortisone.

Patients 7-9 were commenced directly onto fludrocortisone. 
Fig 1a. eGFR (ml/min/1.73 $\left.\mathrm{m}^{2}\right)$ : Before fludrocortisone and at maximum dose of fludrocortisone.

Boxplot note: Horizontal line represents median, rectangle represents first and third quartiles, whiskers represent $2.5^{\text {th }}$ to $97.5^{\text {th }}$ percentiles.

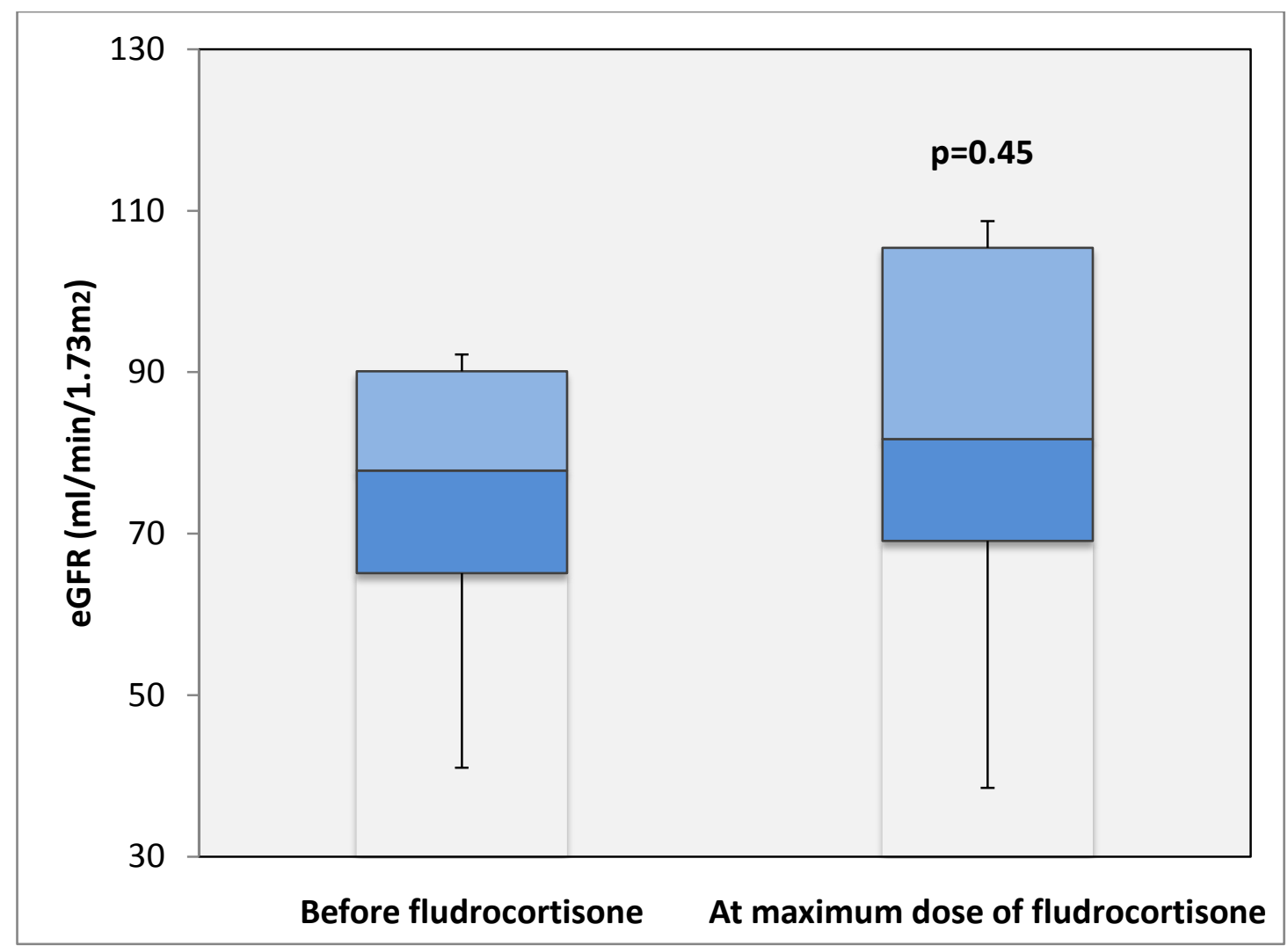


Fig 1b. Serum potassium (mmol/l): Before fludrocortisone and at maximum dose of fludrocortisone.

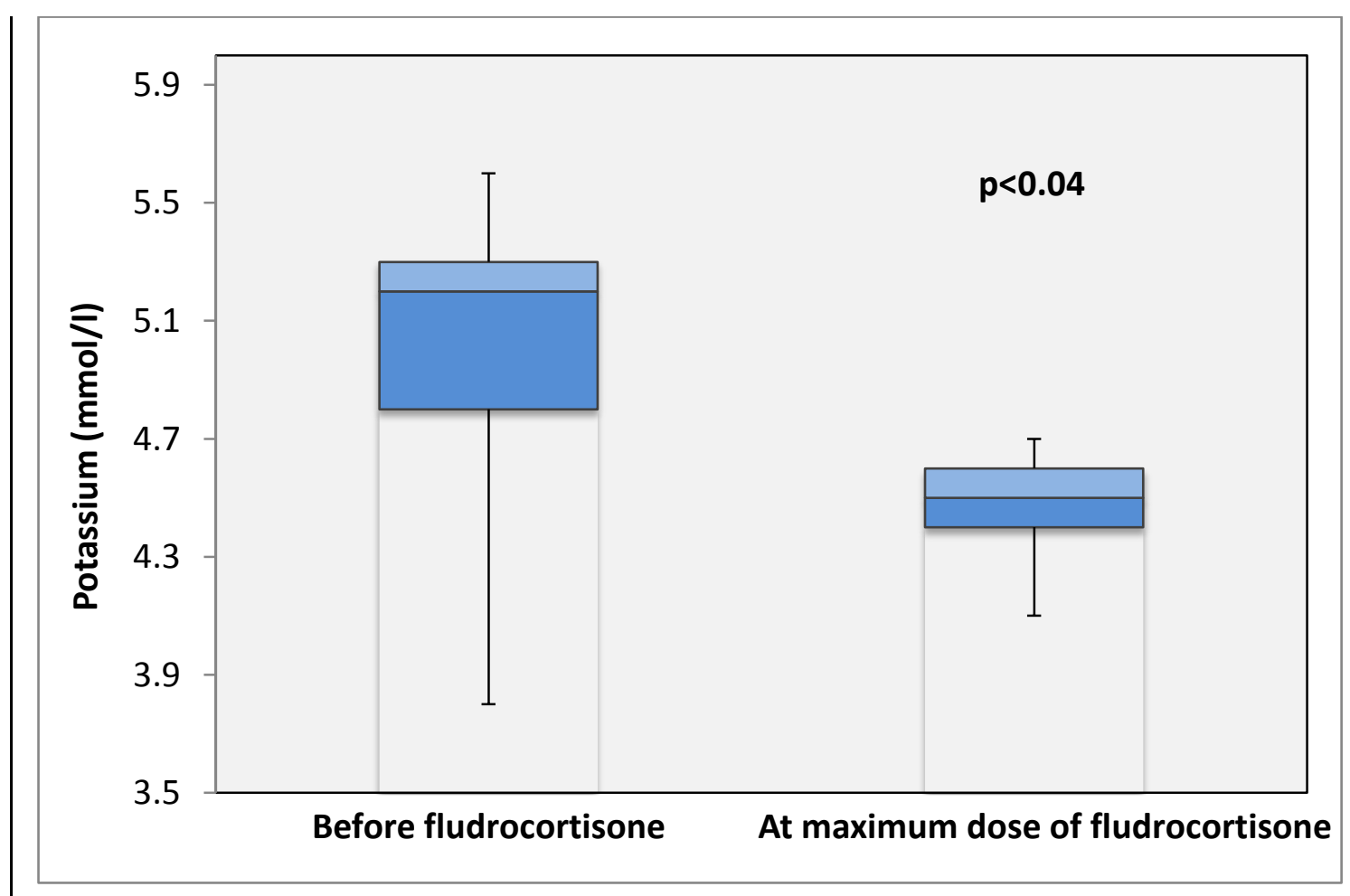


Fig 1c. Systolic blood pressure z-scores: Before fludrocortisone and at maximum dose of fludrocortisone.

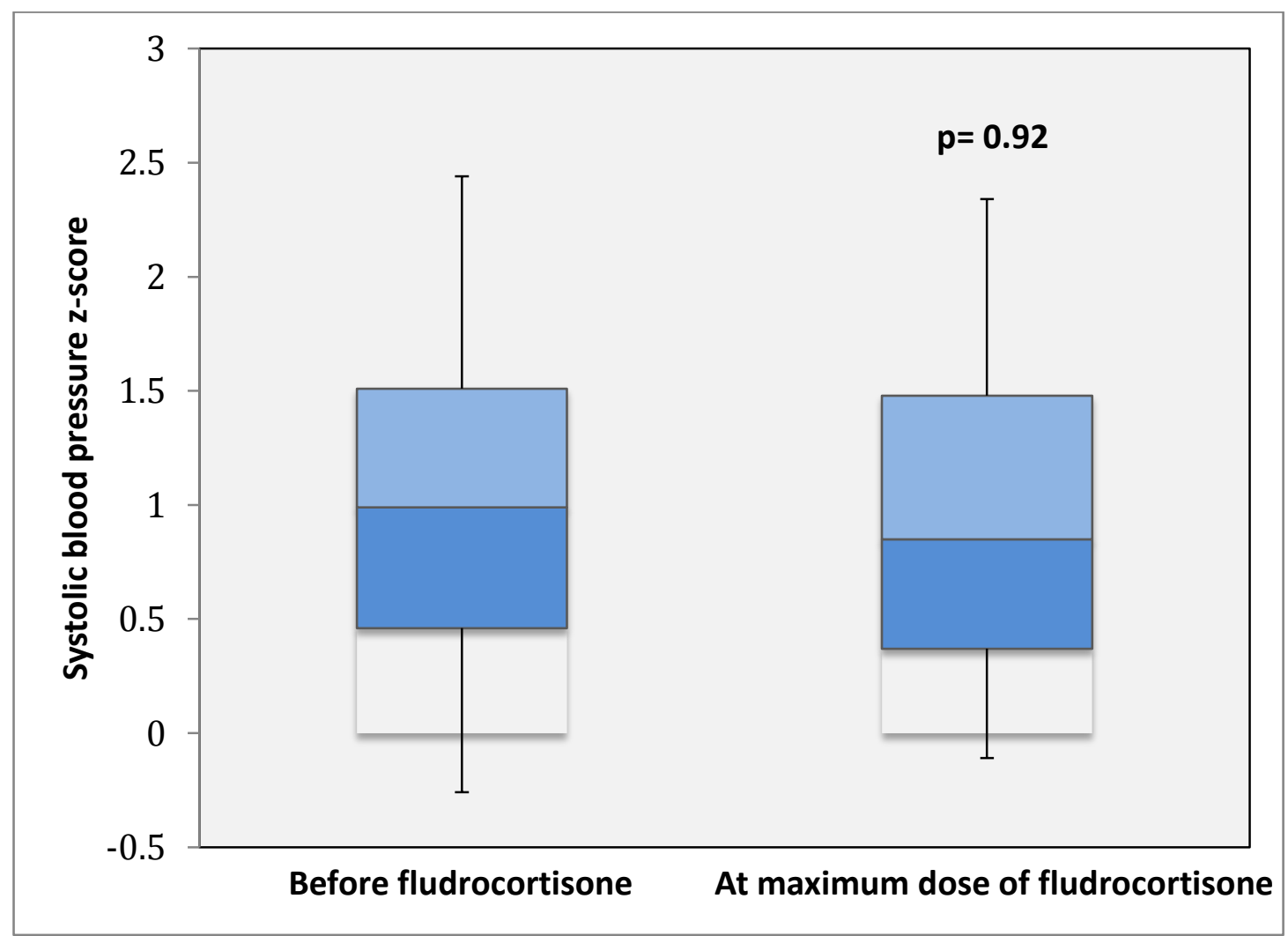




\section{ACKNOWLEDGEMENTS}

The authors would like to acknowledge Susan Burns, Renal Data Manager at the Royal Hospital for Children, Glasgow, for her help in data collection.

\section{CONFLICTS OF INTEREST}

The authors declare no conflicts of interest.

\section{AUTHORS' CONTRIBUTIONS}

Salma R Ali: Contributed to study design, acquisition of data, data analysis and writing of manuscript; Ihab Shaheen: Contributed to the study concept, study design and revision of article; David Young: Contributed to the data analysis; lan Ramage, Heather Maxwell, David A Hughes: Contributed to the study concept; Deepa Athavale: Contributed to study design and revision of article; M Guftar Shaikh: Contributed to the study concept, study design, revision of article and supervision of research. 


\section{REFERENCES}

1. Jones JW, Gruessner RW, Gores PF, Matas AJ. Hypoaldosteronemic hyporeninemic hyperkalaemia after renal transplantation. Transplantation 1993;56:1013-1015.

2. Deppe CE, Heering PJ, Viengchareun S, Grabensee B, Farman N, Lombes M. Cyclosporine a and FK506 inhibit transcriptional activity of the human mineralocorticoid receptor: a cell-based model to investigate partial aldosterone resistance in kidney transplantation. Endocrinology 2002;143:1932-1941.

3. Dagan A, Eisenstein B, Bar-Nathan N, Cleper R, Krause I, Smolkin V, Daidovits M. Tubular and glomerular function in children after renal transplantation. Pediatr Transplant 2005;9:440-4.

4. Heering PJ, Kurschat C, Vo DT, Klein-Vehne N, Fesel K, Ivens K. Aldosterone resistance in kidney transplantation is in part induced by a down-regulation of mineralocorticoid receptor expression. Clin Transplant 2004;18:186-192.

5. Higgins R, Ramaiyan K, Dasgupta T, Kanji H, Fletcher S, Lam F, Kashi H. Hyponatraemia and hyperkalemia are more frequent in renal transplant recipients treated with tacrolimus than with cyclosporin. Further evidence for differences between cyclosporin and tacrolimus nephrotoxicities. Nephrol Dial Transplant 2004;19:444-450.

6. Lin W, Mou L, Tu H, Zhu L, Wang J, Chen J, Hu Y. Clinical analysis of hyperkalaemic renal tubular acidosis caused by calcineurin inhibitors in solid organ transplant recipients. J Clin Pharm Ther 2017;42:122-24. 
7. Dong-Min K, Chung JH, Ho Yoon S, Kim HL. Effect of fludrocortisone acetate on reducing serum potassium levels in patients with end-stage renal disease undergoing haemodialysis. Nephrol Dial Transplant 2007;22:3273-3276.

8. Pavlesa-Kuzmanovska S, Popov Z, Ivanovski O, Ristovska V, Masin-Spasovska J, Rambabova-Busljetic I, Ivanovski N. Cyclosporine nephrotoxicity and early posttransplant hyperkalaaemia in living-donor renal recipients: report of 4 cases. Exp Clin Transplant 2014;12:479-483.

9. Rangel EB, Gomes SA, Machado PG, dos Santos BF, Pestana JO, Pacheco-Silva A, Heilberg IP. Severe hyperkalaemia type 4 renal tubular acidosis after kidney transplantation: a case report. Transplant Proc 2006;38:3112-3115.

10. Sivakumar V, Sriramnaveen P, Krishna C, Manjusha Y, Reddy YS, Sridhar N, Subramanian S. Role of fludrocortisone in the management of tacrolimus-induced hyperkalemia in a renal transplant recipient. Saudi J Kidney Dis Transpl 2014;25:149-151.

11. Dew MA, DiMartini AF, De Vito Dabbs A, Myaskovsky L, Steel J, Unruh M et al. Rates and risk factors for non-adherence to the medical regimen after adult solid organ transplantation. Transplantation 2007;83:858-73.

12. Bacchetta J, Basmaison O, Leclerc AL, Bertholet-Thomas A, Cochat P, Ranchin B. Fludrocortisone as a new tool for managing tubulopathy after pediatric renal transplantation: a series of cases. Pediatr Nephol 2014;29:2061-2064. 
13. National High Blood Pressure Education Program Working Group on High Blood Pressure in Children and Adolescents. The Fourth Report on the diagnosis, evaluation and treatment of high blood pressure in children and adolescents. Pediatrics 2004;114:555-76.

14. Schwartz GJ, Haycock GB, Edelmann CM Jr, Spitzer A. A simple estimate of glomerular filtration rate in children derived from body length and plasma creatinine. Pediatrics 1976;58:259-63.

15. Paediatric formulary committee. British National Formulary for Children (BNFC);2015-2016. P 396. 